\title{
Two-dimensional coordination of guidance and adaptive radiated waveform for interception and rendezvous problems
}

\author{
Alessio Balleri ${ }^{1}$, Alfonso Farina ${ }^{2}$ and Alessio Benavoli ${ }^{3}$ \\ ${ }^{1}$ Centre for Electronic Warfare, Information and Cyber \\ Cranfield University \\ Defence Academy of the UK, Shrivenham, SN6 8LA, UK \\ email: a.balleri@cranfield.ac.uk \\ ${ }^{2}$ Selex ES (Retired) and Visiting Professor at UCL \\ Rome, Italy \\ email: alfonso.farina@outlook.it \\ ${ }^{3}$ Istituto Dalle Molle di Studi sull'Intelligenza Artificiale (IDSIA ) \\ Manno (Lugano), Switzerland \\ email: alessio@idsia.ch
}

\begin{abstract}
We present an algorithm that allows an interceptor aircraft equipped with an airborne radar to meet another air target (the intercepted) by developing a guidance law and automatically adapting and optimising the transmitted waveform on a pulse to pulse basis. The algorithm uses a Kalman filter to predict the relative position and speed of the interceptor with respect to the target. The transmitted waveform is automatically selected based on its ambiguity function and accuracy properties along the approaching path. For each pulse, the interceptor predicts its position and velocity with respect to the target, takes a measurement of range and radial velocity and, with the Kalman filter, refines the relative range and range rate estimates. These are fed into a Linear Quadratic Gaussian ( $L Q G)$ controller that ensures the interceptor reaches the target automatically and successfully with minimum error and with the minimum guidance energy consumption.
\end{abstract}

\section{Introduction}

The task of intercepting a target and/or rendezvous is an important technical challenge that occurs in many defence operations as well as in civilian applications like robotics, Simultaneous Localisation And Map (SLAM) [1] and similar . One of the first papers on optimal guidance for interception and rendezvous dates back to 1971 [2]. In that paper, a sensor on the ground delivers optimal guidance to the interceptor on the basis of the estimated trajectories of the interceptor and the target to reach. The radar transmits a suitable waveform which, however, does not change during the task. Another paper [3] years later develops a procedure to adapt the radiated waveform to minimise the estimation error in a tracking case study. This procedure has been recently named fore-active control.

It is known [4] that a bat looking for a prey (e.g. a moth or a butterfly), during its search, acquisition, tracking and interception phases and along its trajectory to approach the prey, changes 
adaptively the radiated waveform of the calls in order to improve the location of the prey. More precisely, the figures from [5][6] show the time-frequency spectrogram of the radiated calls in the successive phases of the interception. It can be argued that the bat develops an optimal rendezvous trajectory together with an adaptive radiated waveform which improves the location capability of the predator.

In this paper, we take inspiration from the bat and develop an algorithm that guides an airborne radar interceptor towards a target by jointly developing an optimal guidance and automatically adapting and optimising the transmitted waveform on a pulse to pulse basis. We suitably combine the techniques in [2] and [3], namely the optimal linear quadratic Gaussian (LQG) control law and the fore-active control of the radiated waveform. The result we achieve is to emulate what the bat does in its predation. This is an original contribution of [7]. Some preliminary results of the proposed technique were presented in [8].

\section{Theoretical Framework}

We study the case of an interceptor and a target moving with linear kinematics described by a matrix $\mathbf{F}$ and state equations $\mathbf{x}_{\mathbf{f}}(k)$ and $\mathbf{x}_{\mathbf{b}}(k)$ [9]. The trajectory of both the interceptor and the target are subject to Gaussian random perturbations, $\mathbf{G w}_{\mathbf{f}}(k-1)$ and $\mathbf{G w}_{\mathbf{b}}(k-1)$, with zero mean value and covariance matrices $\mathrm{Q}_{\mathrm{f}}$ and $\mathrm{Q}_{\mathrm{b}}$, respectively.

$$
\begin{aligned}
& \mathbf{x}_{\mathbf{f}}(k)=\mathbf{F x}_{\mathbf{f}}(k-1)+\mathbf{G w}_{\mathbf{f}}(k-1) \\
& \mathbf{x}_{\mathbf{b}}(k)=\mathbf{F} \mathbf{x}_{\mathbf{b}}(k-1)+\mathbf{B u}(k-1)+\mathbf{G w}_{\mathbf{b}}(k-1)
\end{aligned}
$$

The term $\mathbf{B u}(k-1)$ is used to model the ability of the interceptor to adapt and control its trajectory at each step. The interceptor is modelled as a controlled system that accepts an input vector $\mathbf{u}(k)$ which is combined linearly with a matrix $\mathbf{B}$ before being applied to the equations describing the target kinematics. We define the difference between the state equations of the interceptor and of the target as the error to reduce to the minimum value at the intercept point

$$
\mathbf{e}(k)=\mathbf{x}_{\mathbf{b}}(k)-\mathbf{x}_{\mathbf{f}}(k)=\mathbf{F e}(k-1)+\mathbf{B u}(k-1)+\mathbf{G w}_{\mathbf{e}}(k-1)
$$

with $\mathbf{G w}_{\mathbf{e}}(k-1)$ being a Gaussian random processes with mean value zero and covariance matrix Q. At each time $k$ the interceptor transmits a waveform to measure its relative distance and radial velocity with respect to the target and uses the measurements to control its trajectory in order to intercept the target with a limited number of radar transmissions $N_{T}$ and with the minimum energy consumption of the interceptor. We assume that the measurement $\mathbf{y}_{\mathbf{e}}(k)$ of the distance and velocity relative to each transmission is a linear function of the error $\mathbf{e}(k)$ as [3]

$$
\mathbf{y}_{\mathbf{e}}(k)=\mathbf{H e}(k)+\boldsymbol{\nu}_{e}\left(k ; \boldsymbol{\theta}_{k}\right)
$$

where $\mathbf{H}$ is the matrix that maps the error into the measurement and $\boldsymbol{\nu}_{e}\left(k ; \boldsymbol{\theta}_{k}\right)$ is a Gaussian random process with mean value zero and a covariance matrix $\mathbf{N}\left(\boldsymbol{\theta}_{k}\right)$. The covariance matrix of each measurement depends on the accuracy of the transmitted waveform $s\left(t ; \boldsymbol{\theta}_{k}\right)$ whose design is fully described by the vector of parameters $\boldsymbol{\theta}_{k}$ that identifies the key waveform properties, 
such as duration, bandwidth and time-frequency curvature. The mathematical expression of the elements of $\boldsymbol{\theta}_{k}$ and the vector length depend on the waveform design. It has been shown in the literature that, when the measurement vector consists only of the measurements of the target range and radial velocity, $\mathbf{N}\left(\boldsymbol{\theta}_{k}\right)$ corresponds to the Cramér-Rao Lower Bound (CRLB) of the estimates of range and radial velocity [10][11][12][13]. The Fisher Information Matrix (FIM) for range and radial velocity in the presence of noise with mean power $N_{0}$ can be expressed as

$$
\mathrm{FIM}=-\left.\mathrm{SNR}\left(\begin{array}{ll}
\frac{4}{c^{2}} \frac{1}{2} \frac{\partial^{2}\left|\chi\left(\tau, \nu ; \boldsymbol{\theta}_{k}\right)\right|^{2}}{\partial \tau^{2}} & \frac{4}{c \lambda} \frac{1}{2} \frac{\partial^{2}\left|\chi\left(\tau, \nu ; \boldsymbol{\theta}_{k}\right)\right|^{2}}{\partial \tau \partial \nu} \\
\frac{4}{c \lambda} \frac{1}{2} \frac{\partial^{2}\left|\chi\left(\tau, \nu ; \boldsymbol{\theta}_{k}\right)\right|^{2}}{\partial \nu \partial \tau} & \frac{4}{\lambda^{2}} \frac{1}{2} \frac{\partial^{2}\left|\chi\left(\tau, \nu ; \boldsymbol{\theta}_{k}\right)\right|^{2}}{\partial \nu^{2}}
\end{array}\right)\right|_{\tau, \nu=0}
$$

where $c$ is the speed of propagation, $\lambda$ is the wavelength at the central angular frequency $w_{0}$, $\mathrm{SNR}=2 E_{s} / N_{0}$ is the Signal to Noise Ratio and $\chi_{k}(\tau, \nu)$ is the normalised narrowband Complex Ambiguity Function (CAF) of the signal $\sqrt{E_{s}} s\left(t ; \boldsymbol{\theta}_{k}\right)$ of energy $E_{s}$ defined as

$$
\chi\left(\tau, \nu ; \boldsymbol{\theta}_{k}\right)=\int_{-\infty}^{\infty} s\left(t ; \boldsymbol{\theta}_{k}\right) s^{*}\left(t+\tau ; \boldsymbol{\theta}_{k}\right) e^{j 2 \pi \nu t} d t
$$

The CRLB is obtained as the inverse of FIM and

$$
\mathbf{N}\left(\boldsymbol{\theta}_{k}\right)=[\mathrm{FIM}]^{-1}
$$

gives the minimum values of variances and covariances of the measurements of range and range rate.

At each time $k$, the interceptor makes a prediction of the estimation error covariance matrix

$$
\mathbf{P}_{k \mid k-1}=\mathbf{F P}_{k-1} \mathbf{F}^{T}+\mathbf{Q}
$$

and then selects the waveform parameters $\boldsymbol{\theta}_{k}$ so to minimise the determinant of the residual matrix

$$
\mathbf{S}_{k}=\mathbf{H P}_{k \mid k-1} \mathbf{H}^{T}+\mathbf{N}\left(\boldsymbol{\theta}_{k}\right)
$$

as described in [3]. The interceptor then produces a pulse, takes a measurement with a waveform of the preselected parameters $\boldsymbol{\theta}_{k}$, and uses the covariance matrix $\mathbf{N}\left(\boldsymbol{\theta}_{k}\right)$ to calculate the Kalman filter gain $\mathbf{K}_{k}$ as

$$
\mathbf{K}_{k}=\mathbf{P}_{k \mid k-1} \mathbf{H}^{T} \mathbf{S}_{k}^{-1}
$$

The Kalman gain is then used to calculate the estimation error covariance matrix at the $\mathrm{k}^{\text {th }}$ step as $\mathbf{P}_{k}=\left(\mathbf{I}-\mathbf{K}_{k} \mathbf{H}\right) \mathbf{P}_{k \mid k-1}$ and an estimate of the error as

$$
\begin{aligned}
& \hat{\mathbf{e}}(k \mid k-1)=\mathbf{F} \hat{\mathbf{e}}(k-1)+\mathbf{B u}(k-1) \\
& \hat{\mathbf{e}}(k)=\hat{\mathbf{e}}(k \mid k-1)+\mathbf{K}_{k}\left[\mathbf{y}_{\mathbf{e}}(k)-\mathbf{H} \hat{\mathbf{e}}(k \mid k-1)\right]
\end{aligned}
$$


Eq. 10 shows that the estimate of the error at the $\mathrm{k}^{\text {th }}$ time step only depends on the control input at the $\mathrm{k}-1^{\text {th }}$ time and this will allow us to select the most appropriate control input at the $\mathrm{k}^{\text {th }}$ time based solely on the estimate of the error.

As previously mentioned, the control task is carried out to ensure the interceptor reaches the target as efficiently as possible. To do this, we define and minimise the cost function

$$
J=E\left\{\mathbf{e}^{T}\left(N_{T}\right) \mathbf{M e}\left(N_{T}\right)+\sum_{k=0}^{N_{T}} \mathbf{u}^{T}(k) \mathbf{R u}(k)\right\}
$$

of the kind of a typical LQG control framework [2]. In Eq. $11 N_{T}$ is the predefined number of transmissions used to intercept the target and $\mathbf{M}$ and $\mathbf{R}$ are two suitable matrices that are applied to the dynamic state error and to the input control signal, respectively. It is worth noting that when $\mathbf{M}$ and $\mathbf{R}$ are identity matrices the cost function is minimised when the error at time $N_{T}$ is minimised and when the energy of the input signal $\mathbf{u}(k)$ is also minimised. The solution of Eq. 11 is well known in the literature (e.g. see [2]) and it is such that the control input signal at the time $k$ is a function of the estimate of the error at the time $k$ and of a matrix $\mathbf{L}_{k}$

$$
\mathbf{u}(k)=-\mathbf{L}_{k} \hat{\mathbf{e}}(k)
$$

where

$$
\mathbf{L}_{k+1}=\left(\mathbf{B}^{T} \mathbf{U}_{k+1} \mathbf{B}+\mathbf{R}\right)^{-1} \mathbf{B}^{T} \mathbf{U}_{k+1} \mathbf{F}
$$

and

$$
\mathbf{U}_{k}=\mathbf{F}^{T}\left(\mathbf{U}_{k+1}-\mathbf{U}_{k+1} \mathbf{B}\left(\mathbf{B}^{T} \mathbf{U}_{k+1} \mathbf{B}+\mathbf{R}\right)^{-1} \mathbf{B}^{T} \mathbf{U}_{k+1}\right) \mathbf{F}, \text { with } \mathbf{U}_{N_{T}}=\mathbf{M}
$$

We note that the interleave between fore-active control and LQG control is new and represents one of the contributions of [7].

\subsection{Gaussian Linear Chirp}

We limit the study to Linear Frequency Modulated chirps (LFM) with a Gaussian amplitude modulation of the form

$$
s\left(t ; \boldsymbol{\theta}_{k}\right)=\left(\frac{1}{\pi \lambda_{G}^{2}}\right)^{\frac{1}{4}} e^{-\frac{t^{2}}{2 \lambda_{G}^{2}}} e^{j b_{G} t^{2}} e^{j 2 \pi f_{0} t}
$$

whose design depends solely on the parameters $\lambda_{G}$ and $b_{G}$, that is $\boldsymbol{\theta}_{k}=\left[b_{G} \lambda_{G}\right]^{T}$. Selecting and diversifying these parameters results in waveforms with a different time duration $T=2 \lambda_{G}$ and bandwidth $B=b_{G} T / \pi$. The use of a Gaussian linear chirp simplifies the analysis because the covariance matrix of the estimators of range and range rate is known in the literature (e.g see [3] and [14]) and can be expressed as

$$
\mathbf{N}\left(\boldsymbol{\theta}_{k}\right)=\left(\begin{array}{rr}
\frac{c^{2} \lambda_{G}^{2}}{2 \mathrm{SNR}} & -\frac{c \lambda \lambda_{G}^{2} b_{G}}{2 \pi \mathrm{NNR}} \\
-\frac{c \lambda \lambda_{G}^{2} b_{G}}{2 \pi \mathrm{SNR}} & \frac{\lambda^{2}}{4 \pi^{2} \mathrm{SNR}}\left(\frac{1}{2 \lambda_{G}^{2}}+2 \lambda_{G}^{2} b_{G}^{2}\right)
\end{array}\right)
$$


It can be easily shown that the determinant of the FIM is equal to $\operatorname{SNR}^{2} \frac{16 \pi^{2}}{c^{2} \lambda^{2}}$ and does not depend on the parameters $b_{G}$ and $\lambda_{G}$ [3]. This will significantly simplify the calculations of the optimal $\boldsymbol{\theta}_{k}$.

\section{Two-dimensional Case Study}

We study the case of a static target and an interceptor that moves on a two-dimensional plane. The state variables are expressed in polar coordinates and consist of the range between the target and the interceptor $\rho$, the radial velocity $\dot{\rho}$ and the relative angle $\theta$. Although, in practice, problems of tracking in 2D and 3D are treated in the Cartesian coordinates with the use of non-linear equations, using polar coordinates allows the treatment of the problem tackled in this paper with linear equations. The use of linear equation is of particular importance as it guarantees the convergence of the LGQ control. A noisy perturbation is applied to the component representing the acceleration and to the angular velocity of the interceptor. To represent this scenario, we define the matrices $\mathbf{F}$ and $\mathbf{G}$ as

$$
\mathbf{F}=\left(\begin{array}{ccc}
1 & T_{s} & 0 \\
0 & 1 & 0 \\
0 & 0 & 1
\end{array}\right)
$$

and

$$
\mathbf{G}=\left(\begin{array}{ll}
0 & 0 \\
1 & 0 \\
0 & 1
\end{array}\right)
$$

The resulting interceptor state equations in absence of control are

$$
\left\{\begin{array}{l}
\rho(k)=\rho(k-1)+\dot{\rho}(k-1) T_{s} \\
\dot{\rho}(k)=\dot{\rho}(k-1)+a \\
\theta(k)=\theta(k-1)+b
\end{array}\right.
$$

where $T_{s}$ is the radar scan period and $a$ and $b$ are two independent Gaussian processes with zero mean value and variance $\sigma_{a}^{2}$ and $\sigma_{b}^{2}$, respectively. ${ }^{1}$

The sensor measures the distance between the interceptor and the target, their relative radial velocity and the relative angle. The matrix $\mathbf{H}$ is defined as

$$
\mathbf{H}=\left(\begin{array}{lll}
1 & 0 & 0 \\
0 & 1 & 0 \\
0 & 0 & 1
\end{array}\right)
$$

\footnotetext{
${ }^{1}$ Here, we consider the case of one pulse per scan and hence the scan period corresponds to the Pulse Repetition Interval (PRI) of the radar.
} 
The covariance matrix of the measurement is obtained by modifying Eq.16 so to account for the measurement of the angle as

$$
\hat{\mathbf{N}}\left(\boldsymbol{\theta}_{k}\right)=\left(\begin{array}{rrr}
\frac{c^{2} \lambda_{G}^{2}}{2 \mathrm{SNR}} & -\frac{c \lambda \lambda_{G}^{2} b_{G}}{2 \pi \mathrm{SNR}} & 0 \\
-\frac{c \lambda \lambda_{G}^{2} b_{G}}{2 \pi \mathrm{SNR}} & \frac{\lambda^{2}}{4 \pi^{2} \mathrm{SNR}}\left(\frac{1}{2 \lambda_{G}^{2}}+2 \lambda_{G}^{2} b_{G}^{2}\right) & 0 \\
0 & 0 & \sigma_{\theta}^{2}
\end{array}\right)
$$

under the assumption that the measurement of the angle is statistically independent of the measurements of the range and the radial velocity. The waveform parameters of the Gaussian linear chirp $\boldsymbol{\theta}_{k}=\left[\begin{array}{ll}b_{G} & \lambda_{G}\end{array}\right]^{T}$ at the $k$-th time step can be estimated from the element $p_{i j}$ of the matrix $\mathbf{P}_{k \mid k-1}$ so to minimise the determinant of the matrix $\mathbf{S}_{k}$. After some simple algebra it can be shown that the determinant of $\mathbf{S}_{k}$ is minimised for

$$
b_{G}=\frac{\gamma w_{0}}{4 \beta}
$$

and

$$
\lambda_{G}=\left(\frac{\beta}{\delta}\right)^{0.25}
$$

with

$$
\left\{\begin{array}{l}
\hat{p}=p_{3,3}+\sigma_{\theta}^{2} \\
\alpha=\hat{p} p_{2,2}-p_{3,2} p_{2,3} \\
\beta=\hat{p} p_{1,1}-p_{3,1} p_{1,3} \\
\gamma=p_{3,2} p_{1,3}-2 \hat{p} p_{1,2}+p_{3,1} p_{2,3} \\
\delta=w_{0}^{2} \alpha-2 \gamma w_{0} b_{G}+4 b_{G}^{2} \beta
\end{array}\right.
$$

\section{Simulation Results}

Simulations are carried out for the case of an interceptor that for $k=0$ is at $10 \mathrm{~km}$ from the target moving with a relative radial velocity of $-50 \mathrm{~m} / \mathrm{s}$ and with a relative angle of 0 degrees $\left(\mathbf{e}(0)=\left[10^{4}-500\right]^{\mathrm{T}}\right)$. The filter is initialised with $\hat{\mathbf{e}}(0)$ being a realisation of a Gaussian random variable with mean value $\mathbf{e}(0)$ and covariance matrix $\mathbf{P}_{0}$. This is a diagonal matrix with all the elements on the diagonal equal to $10^{6}$. The covariance matrix $\mathbf{P}_{0}$ is very large in order to guarantee the filter accepts the first set of measurements which typically, for a single radar pulse, are characterised by a very large covariance matrix due to the low accuracy of a single chirp. The SNR at time zero is set to $20 \mathrm{~dB}$ and the parameter $\sigma_{\theta}$ is fixed to 0.017 as a representative beamwidth of a $0.5 \mathrm{~m}$ aperture at $35 \mathrm{GHz}$. The control is set to reach the target within $N_{T}=300$ transmissions and the scan rate $T_{s}$ is equal to $1 \mathrm{~s}$. Figure 1 shows the rangerange rate diagram for the case with constraints on both the pulse width and the bandwidth of the chirp. The pulse width cannot assume values below $1 \mu \mathrm{sec}$ and cannot assume values that would generate eclipsing. The eclipsing limit is calculated by using the estimate of the range between the target and the interceptor at each step $k$ as $2 \rho_{k} / c$. The bandwidth cannot reach values below $20 \mathrm{MHz}$, corresponding to a minimum range resolution equal to $7.5 \mathrm{~m}$. Results show that LQG control converges as expected and that the interceptor reaches the target within the predefined 


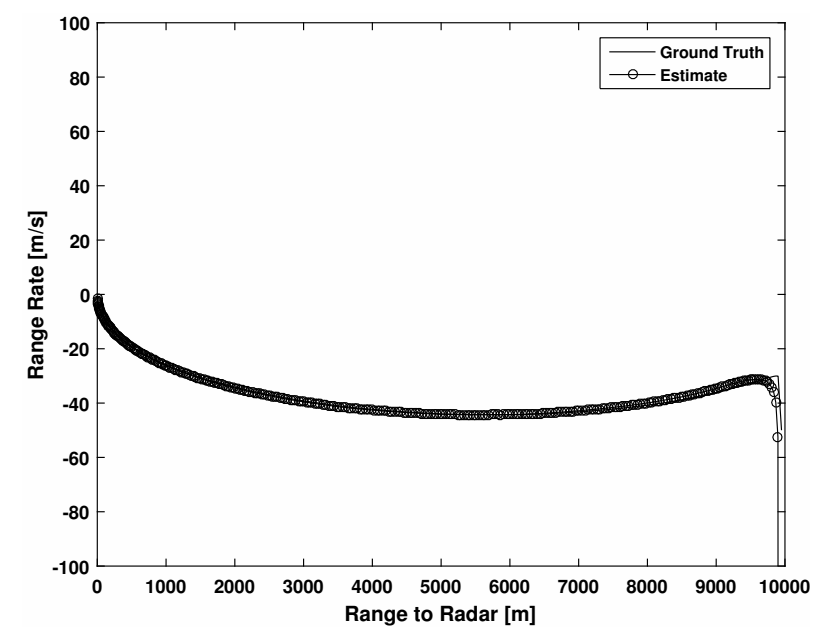

Figure 1: Range- Range Rate diagram for the case with constraints applied to the chirp parameters.

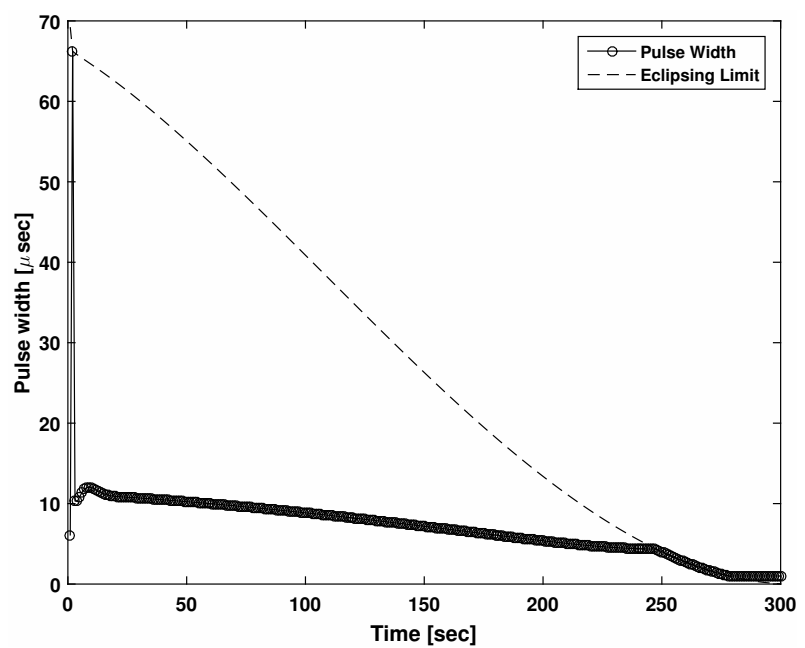

Figure 2: Pulse width of each transmission for the case with constraints applied to the chirp parameters.

$N_{T}$ transmissions. The error at the beginning of the sequence is reasonable because, due to the applied constraints, the chirp parameters assume values that result in a lower measurement covariance matrix starting from the very first transmissions. Results in Figure 2 show that the pulse duration assumes values between $5 \mu$ s up to about $15 \mu \mathrm{s}$. The bandwidth remains constant throughout the sequence at $20 \mathrm{MHz}$.

Figure 3 shows the AF of the $1^{\text {st }}, 75^{\text {th }}, 225^{\text {th }}$ and $300^{\text {th }}$ transmitted waveforms. Results show that the wedge of the AF rotates anti-clockwise along the trajectory. When the interceptor approaches the target, the bandwidth does not change significantly and the range resolution remains constant. However, as the pulse duration becomes shorter the Doppler resolution decreases. The waveform is Doppler tolerant throughout the mission ${ }^{2}$, that is the output of the matched-filter remains high in the presence of a Doppler mismatch.

\footnotetext{
${ }^{2}$ Doppler tolerance is a characteristic of linear chirps when the narrowband approximation is satisfied [14].
} 

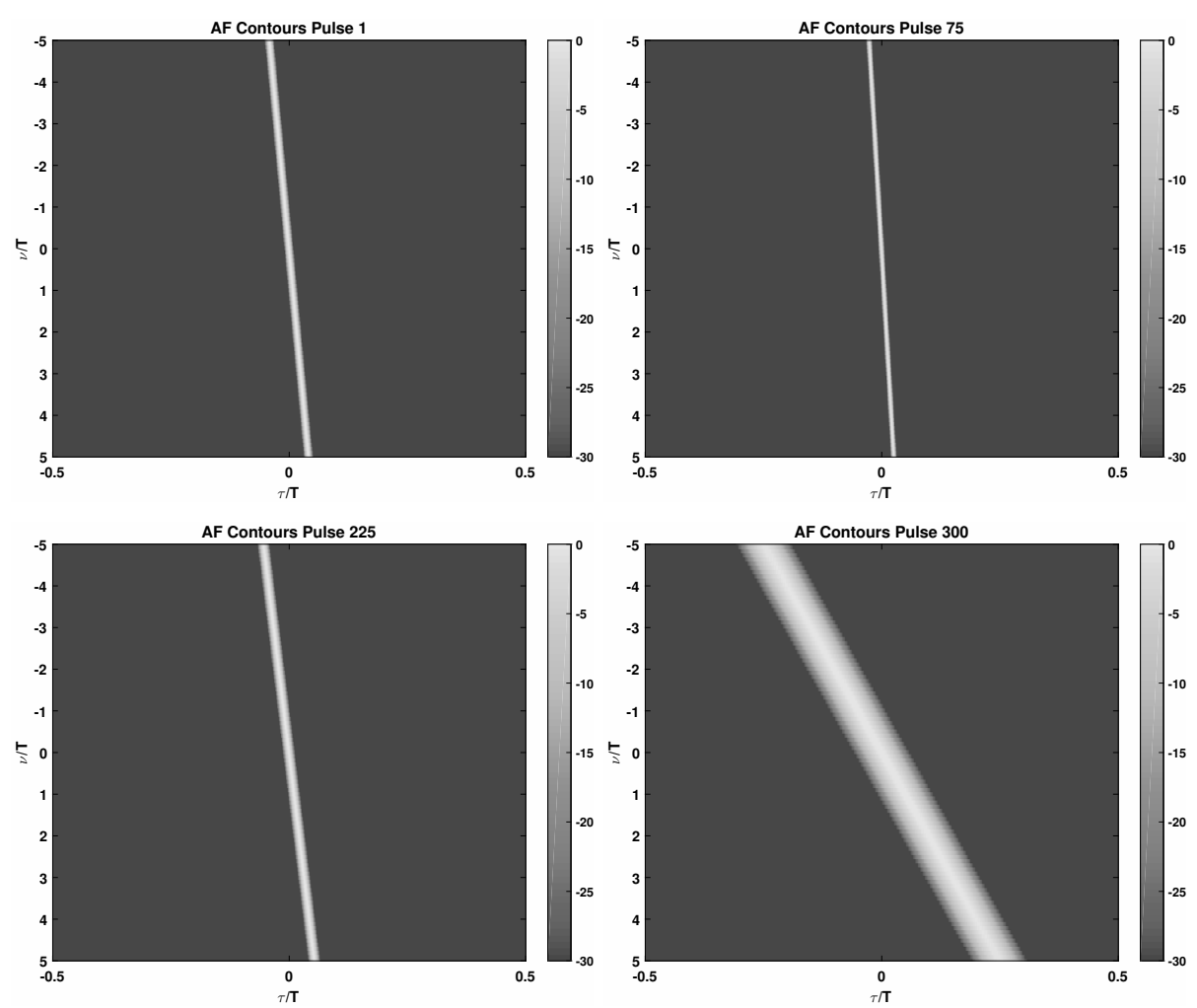

Figure 3: Contours of the AF of the $1^{\text {st }}, 75^{\text {th }}, 225^{\text {th }}$ and $300^{\text {th }}$ transmitted waveforms for the case with constraints applied to the chirp parameters.

\section{Conclusions and Future Work}

In this paper, we have presented an algorithm that allows an interceptor aircraft with an onboard radar to adapt its trajectory in order to intercept a target and to automatically optimising the transmitted waveform on a pulse to pulse basis. To achieve this, we have suitably combined two techniques, namely the optimal Linear Quadratic Gaussian (LQG) control law and the fore-active control of the radiated waveform. Simulation results show that the interceptor can successfully reach the target within the predefined number of transmissions and automatically adapt the waveform during the mission. Future work will look at using different types of waveform designs and different optimisation criteria.

\section{References}

[1] M. W. M. G. Dissanayake, P. Newman, S. Clark, H. F. Durrant-Whyte, and M. Csorba, "A solution to the simultaneous localization and map building (SLAM) problem," IEEE Transactions on Robotics and Automation, vol. 17, no. 3, pp. 229-241, Jun 2001.

[2] M. Athans, "On optimal allocation and guidance laws for linear lnterception and rendezvous problems," IEEE Transactions on Aerospace and Electronic Systems, vol. AES-7, no. 5, pp. 843-853, Sept 1971. 
[3] D. Kershaw and R. Evans, "Optimal waveform selection for tracking systems," IEEE Transactions on Information Theory, vol. 40, no. 5, pp. 1536-1550, Sep 1994.

[4] C. Baker, H. Griffiths, and A. Balleri, "Biologically inspired waveform diversity (in Waveform Design and Diversity for Advanced Radar Systems)," Institution of Engineering and Technology, Series on Radar, Sonar, Navigation and Avionics, pp. 149-172, 2012.

[5] P. Flandrin, Animal Sonar: Processes and Performance. Boston, MA: Springer US, 1988, ch. Time-Frequency Processing of Bat Sonar Signals, pp. 797-802.

[6] F. Hlawatsch and G. F. Boudreaux-Bartels, "Linear and quadratic time-frequency signal representations," IEEE Signal Processing Magazine, vol. 9, no. 2, pp. 21-67, April 1992.

[7] A. Balleri, A. Farina, and A. Benavoli, "Coordination of optimal guidance law and adaptive radiated waveform for interception and rendezvous problems," accepted for IET Radar, Sonar and Navigation, December 2016.

[8] A. Farina, "Cognitive Radar Signal Processing," Key Note Speech, IET International Radar Conference, Hangzhou, China, 14-16 Oct. 2015.

[9] R. Kalman, "Mathematical description of linear dynamical systems," Journal of the Society for Industrial and Applied Mathematics, Series A: Control, vol. 1, no. 2, pp. 152-192, 1963.

[10] H. L. Van Trees, Detection, estimation and modulation theory, Part III. Radar-Sonar signal processing and Gaussian signals in noise. Wiley, 2001.

[11] C. E. Cook and M. Bernfeld, Radar Signals:an introduction to theory and application. Artech House, 1987.

[12] E. Kelly, "The radar measurement of range, velocity and acceleration," IRE Transactions on Military Electronics, vol. MIL-5, no. 2, pp. 51-57, April 1961.

[13] A. Dogandzic and A. Nehorai, "Cramer-Rao bounds for estimating range, velocity, and direction with an active array," IEEE Transactions on Signal Processing, vol. 49, no. 6, pp. 1122-1137, Jun 2001.

[14] A. Balleri and A. Farina, "Ambiguity function and accuracy of the hyperbolic chirp: comparison with the linear chirp," IET Radar, Sonar and Navigation, Online May 2016. 